\title{
Evaluation of Concentration Dependence of $\chi$-Parameter, Flory Temperature and Entropy Parameter for Polymer-Solvent System from Their Critical Solution Temperature and Concentration Data
}

\author{
Kenji KAMIDE, Shigenobu MAtsudA, and Masatoshi SAITO \\ Textile Research Laboratory, Asahi Chemical Industry Company, Ltd., \\ 11-7, Hacchonawate, Takatsuki, Osaka 569, Japan
}

(Received November 8, 1984)

\begin{abstract}
The critical polymer volume fraction $v_{\mathrm{p}}^{\mathrm{c}}$ and the critical solution temperature $T_{\mathrm{c}}$ were estimated from the threshold cloud point (tcp) for polystyrene (PS) and polyethylene (PE) solutions in literature. The critical points thus obtained, together with those directly determined in literature, of PS in ten solvents and PE in sixteen solvents were analyzed, according to the ShultzFlory (SF), Koningsveld et al. (KKS), and Kamide-Matsuda (KM) methods, to evaluate the Flory temperature $\theta$ and the entropy parameter $\psi$. The concentration dependence parameters $p_{1}$ and $p_{2}$ of the $\chi$-parameter, as given by $\chi=\chi_{0}\left(1+p_{1} v_{\mathrm{p}}+p_{2} v_{\mathrm{p}}^{2}\right)\left(\chi_{0}\right.$, a concentration-independent parameter, $v_{\mathrm{p}}$, polymer volume fraction) were determined by KKS and KM methods, which gave almost the same values of $p_{1}$ and $p_{2}$. Except for few solvents, $p_{1}$ value for PS solutions can be regarded as constant, which is near to $2 / 3$, theoretically predicted when $A_{2}=A_{3}=0$ at $\theta$ ( $A_{2}$ and $A_{3}$, second and third virial coefficients). For lower critical solution point (LCSP), $p_{2}$ is always negative, except for PS/ cyclopentane and for upper critical solution point (UCSP) $p_{2}$ is positive. The very wide variation of $p_{1}$ and $p_{2}$ with solvents was observed for PE. Application of KM method to the most reliable data on PE/diphenylether by Koningsveld $e t$ al. led to $p_{1}=0.61$, which is not so far from the theoretical value. $\theta$, obtained by three methods, is practically independent of the method used. $\psi$ estimated by KM method coincides with that by KKS method for a given polymer-solvent system. $\psi$ was found to be positive for UCSP and negative for LCSP, suggesting that the polymer solution should be athermal at a temperature between UCSP and LCSP. The effect of solvent nature on $p_{1}, p_{2}, \theta$, and $\psi$ are discussed in some detail.
\end{abstract}

KEY WORDS Critical Polymer Volume Fraction / Critical Solution Temperature / Threshold Cloud Point / Polystyrene / Polyethylene / Flory Theta Temperature / Entropy Parameter / Concentration Dependence Parameter / $\chi$-Parameter /

Koningsveld et al. $(\mathrm{KKS})^{1}$ and Kamide and Matsuda $(\mathrm{KM})^{2}$ proposed methods for estimating the concentration dependence parameters $p_{1}$ and $p_{2}$ of the polymer-solvent interaction parameter $\chi$ as given by

$$
\chi=\chi_{0}\left(1+p_{1} v_{\mathrm{p}}+p_{2} v_{\mathrm{p}}^{2}\right)
$$

from the critical solution temperature $T_{\mathrm{c}}$ and the critical polymer volume fraction $v_{\mathrm{p}}^{\mathrm{c}}$ of the polymer solutions. Here, $\chi_{0}$ is the concentration independent parameter and $v_{\mathrm{p}}$ is the polymer volume fraction. The concentration dependence of $\chi$-parameter can also be, from a theoretical point of view, determined by the osmotic pressure, vapor pressure, isothermal distillation, ultracentrifuge, phase equilibrium (the two phase volume ratio $R$, the partition coefficient $\sigma$, the polydispersity of the polymers in two phases etc.) and the cloud point. ${ }^{3}$ It should be noted that except for the phase equilibrium and cloud point, all methods are limited experimentally to a relatively 
lower concentration range and do not enable us to evaluate $p_{2}$ accurately. The phase equilibrium method is applicable up to a concentrated solution range, but the experimental accuracy is not high enough to estimate $p_{2}$. The remaining two methods (the critical and cloud points) are the most suitable for determining $p_{1}$ and $p_{2}$. Kamide and Matsuda have shown for polystyrene/cyclohexane system $p_{1}=0.643$ and $p_{2}=0.200$ from the cloud point curve data of themselves ${ }^{3}$ and $p_{1}=0.642$ and $p_{2}=0.190$ from literature data on the critical point using $\mathrm{KM}$ method. ${ }^{2}$ Using $p_{1}$ and $p_{2}$ paramters thus estimated and the critical solution data, the Flory theta temperature $\theta$ and the entropy parameter at $\theta, \psi$ can also be determined. ${ }^{1,2}$

Kamide and Matsuda $^{2}$ applied these two methods to the systems of polystyrene(PS)/ cyclohexane $(\mathrm{CH})$ and $\mathrm{PS} /$ methylcyclohexane $(\mathrm{MCH})$, concluding the two methods to give similar results on $p_{1}, p_{2}, \theta$, and $\psi$ for a given system and that $\psi$ values obtained by these methods are remarkably smaller than those by the Shultz and Flory (SF) ${ }^{4}$ method hithertofore widely utilized, lying in the range of variation of $\psi$ values evaluated from the temperature dependence of the second virial coefficient $A_{2} \cdot{ }^{5}$ In other words, Kamide and Matsuda clarified the unconditioned superiority of the KKS and KM methods over the SF method, in which $p_{1}=p_{2}=0$ was assumed. In this article, as an extension of the previous study, ${ }^{2}$ an attempt is made by applying the above mentioned methods (i.e., KKS, KM, and $\mathrm{SF})$ to the literature data on the critical points of the polymer solutions in a very systematic manner to compare the reliability of these methods and disclose, if possible, the dependence of $p_{1}, p_{2}, \theta$, and $\psi$ parameters on the solvent nature.

\section{THEORETICAL BACKGROUND}

Equation 1 can be generalized in the form: ${ }^{6}$

$$
\chi=\chi_{0}\left(1+\sum_{i=1}^{n} p_{i} v_{\mathrm{p}}^{i}\right)
$$

with

$$
\begin{aligned}
\chi_{0} & =\chi_{00}\left(1+k^{\prime} / X_{n}\right) \\
& =(a+b / T)\left\{1+\frac{k_{0}}{X_{n}}\left(1-\frac{\theta}{T}\right)\right\}
\end{aligned}
$$

Here, $\chi_{00}$ is a parameter independent of $v_{\mathrm{p}}$ and of the molar volume ratio of $j$-th component of multicomponent polymers to the solvent $X_{j}$ $(j=1,2, \cdots, m: m=$ total number of the components constituting the polymer), $k^{\prime}$ and $p_{i}$, the molecular weight- and concentrationdependence parameters, both independent of $v_{\mathrm{p}}$ and $X_{j} . T$ is Kelvin temperature, $a, b$, and $k_{0}$ are coefficients independent of $X_{n}, v_{\mathrm{p}}$, and $T$.

The chemical potential of the solvent $\Delta \mu_{0}$, in the Flory-Huggins polymer solution theory, ${ }^{7}$ can be given by

$$
\begin{aligned}
\Delta \mu_{0}= & R T\left\{\ln \left(1-v_{\mathrm{p}}\right)+\left(1-\frac{1}{X_{n}}\right) v_{\mathrm{p}}\right. \\
& \left.+\chi_{00}\left(1+\frac{k^{\prime}}{X_{n}}\right)\left(1+\sum_{i=1}^{n} p_{i} v_{\mathrm{p}}^{i}\right) v_{\mathrm{p}}^{2}\right\}
\end{aligned}
$$

Here, $R$ is the gas constant. The chemical potential of $X_{j}$-mer, $\Delta \mu_{j}$, which satisfies the Gibbs-Duhem relation with $\Delta \mu_{0}$ in eq 4 , is

$$
\begin{aligned}
\Delta \mu_{j}= & R T\left[\ln v_{j}-\left(X_{j}-1\right)+X_{j}\left(1-\frac{1}{X_{n}}\right) v_{\mathrm{p}}\right. \\
+ & X_{j}\left(1-v_{\mathrm{p}}\right)^{2} \chi_{00}\left[\left(1+\frac{k^{\prime}}{X_{n}}\right)\right. \\
& \times\left\{1+\sum_{i=1}^{n} \frac{p_{i}}{i+1}\left(\sum_{q=0}^{i}(q+1) v_{\mathrm{p}}^{q}\right)\right\} \\
+ & k^{\prime}\left(\frac{1}{X_{j}}-\frac{1}{X_{n}}\right)\left\{\frac{1}{1-v_{\mathrm{p}}}\right. \\
& \left.\left.\left.+\sum_{i=1}^{n} \frac{p_{i}}{i+1}\left(\sum_{q=0}^{i} \frac{v_{\mathrm{p}}^{q}}{1-v_{\mathrm{p}}}\right)\right\}\right]\right]
\end{aligned}
$$

The Gibbs free enregy $\Delta G^{\prime}$ per unit volume of the solution

$$
\Delta G^{\prime}=\left(1-v_{\mathrm{p}}\right)\left(\frac{\Delta \mu_{0}}{V_{0}}\right)+\sum_{j=1}^{m} v_{j}\left(\frac{\Delta \mu_{j}}{X_{j} V_{0}}\right)
$$


can be calculated from eq 4 and 5 . Here, $V_{0}$ is the solvent molar volume and $v_{j}$ is the volume fraction of $X_{j}$-mer. Substitution of eq 6 into the equation for the spinodal and that for the condition, under which the equilibrium is neutral, gives ${ }^{8}$

$$
\begin{gathered}
\frac{1}{X_{w} v_{\mathrm{p}}}+\frac{1}{1-v_{\mathrm{p}}}+\chi_{00}\left\{1+k^{\prime}\left(1+\frac{1}{X_{n}}-\frac{X_{w}}{X_{n}}\right)\right\} \\
\times\left\{2+\sum_{i=1}^{n} p_{i}(i+2) v_{\mathrm{p}}^{i}\right\}=0 \\
\frac{1}{\left(1-v_{\mathrm{p}}\right)^{2}}-\frac{X_{z}}{X_{w}^{2} v_{\mathrm{p}}^{2}}-\chi_{00}\left\{1+k^{\prime}\left(1+\frac{1}{X_{n}}-\frac{X_{w}}{X_{n}}\right)\right\} \\
\times\left\{\sum_{i=1}^{n} p_{i}(i+2) v_{\mathrm{p}}^{i-1}\right\}=0
\end{gathered}
$$

where $X_{w}$ and $X_{z}$ are the weight- and $z$-average $X$. At the critical point, eq 7 and 8 should be satisfied simultaneously. $\chi_{00}$ at the critical point $\left(\chi_{00}^{\mathrm{c}}\right)$ is related to $T_{\mathrm{c}}, \theta$, and $\psi$ through the relation, ${ }^{8}$

$$
\chi_{00}^{\mathrm{c}}=\left(\frac{1}{2}-\psi\right)+\frac{\theta \psi}{T_{\mathrm{c}}}
$$

with

$$
\psi=\frac{1}{2}-\chi_{00, \mathrm{~s}}
$$

and

$$
\theta=\chi_{00, \mathrm{~h}} T_{\mathrm{c}} /\left(1 / 2-\chi_{00, \mathrm{~s}}\right)
$$

$\chi_{00, \mathrm{~s}}$ and $\chi_{00, \mathrm{~h}}$ are entropy and enthalpy terms of $\chi_{00}, \quad$ respectively (i.e., $\chi_{00}=$ $\left.\chi_{00, \mathrm{~s}}+\chi_{00, \mathrm{~h}}\right)$.

Substituting eq 9 into eq 3 , we obtain

$$
\chi_{0}^{c}=a^{\prime}+\frac{b^{\prime}}{T_{c}}+\frac{c^{\prime}}{T_{c}^{2}}
$$

with

$$
\begin{aligned}
& a^{\prime}=\left(\frac{1}{2}-\psi\right)\left(1+\frac{k_{0}}{X_{n}}\right) \\
& b^{\prime}=\theta \psi+\theta\left(\psi-\frac{1}{4}\right) \frac{2 k_{0}}{X_{n}}
\end{aligned}
$$

$$
c^{\prime}=-\left(\frac{k_{0}}{X_{n}}\right) \psi \theta^{2}
$$

The effect of the molecular weight dependence of $\chi$-parameter on the critical point can be considered to be relatively small and then, the critical point is expected to be explained in terms of the concentration-dependence parameters alone. In this case $\left(k^{\prime}=0, k_{0}=0\right)$, eq 9 reduces to

$$
\frac{1}{T_{\mathrm{c}}}=\frac{\chi_{0}^{\mathrm{c}}}{\theta \psi}+\frac{1}{\theta}\left(1-\frac{1}{2 \psi}\right)
$$

Assuming $p_{\mathrm{i}}(i=1,2, \cdots, m)=0$ and $X_{w}=X_{z}$, eq 16 coincides with the equation of Shultz and Flory. ${ }^{4}$

$$
\frac{1}{T_{c}}=\frac{1}{\theta \psi}\left(\frac{1}{2 X_{w}}+\frac{1}{X_{w}^{1 / 2}}\right)+\frac{1}{\theta}
$$

$\Delta \mu_{0}$ in eq 4 can be rewritten by expanding the term $\ln \left(1-v_{\mathrm{p}}\right)$ in Taylor expansion form, and by expressing $v_{\mathrm{p}}$ by the weight concentration, by

$$
\begin{aligned}
\Delta \mu_{0}= & -R T c V_{0}\left\{\frac{1}{M_{n}}+\frac{\bar{v}^{2}}{V_{0}}\left(\frac{1}{2}-\chi_{0}\right) c\right. \\
& \left.+\sum_{i=1}^{n} \frac{\bar{v}^{i+2}}{V_{0}}\left(\frac{1}{i+2}-\chi_{0} p_{i}\right) c^{i+1}\right\}
\end{aligned}
$$

Here, $\bar{v}$ is the polymer specific volume, and $M_{n}$, the number-average molecular weight.

Osmotic pressure $\pi$ is given in virial expansion form,

$$
\begin{aligned}
\pi & =-\frac{\Delta \mu_{0}}{V_{0}} \\
& =R T c\left(\frac{1}{M_{n}}+A_{2} c+A_{3} c^{2}+A_{4} c^{3}+\cdots\right)
\end{aligned}
$$

where $A_{2}, A_{3}, A_{4}, \cdots$ are the second, third, forth, $\cdots$ virial coefficients, and are given using eq 18 as follows,

$$
A_{2}=\frac{\bar{v}^{2}}{V_{0}}\left(\frac{1}{2}-\chi_{o}\right)
$$

and 
$A_{i+2}=\frac{\bar{v}^{i+2}}{V_{0}}\left(\frac{1}{i+1}-\chi_{0} p_{i}\right) \quad(i=1,2, \cdots, n)$

If we can assume that $A_{2}=A_{3}=A_{4}=\cdots=0$ at $T=\theta$ for UCSP, we obtain

$$
\begin{aligned}
& \chi_{0}=\frac{1}{2}, \\
& p_{1}=\frac{2}{3}, \quad p_{2}=\frac{2}{4}, \quad p_{3}=\frac{2}{5}, \cdots, p_{n}=\frac{2}{n+2}
\end{aligned}
$$

These are the theoretically predicted values for $\chi_{0}$ and $p_{j}(j=1, \cdots, n)$. In LCSP region, $p_{1}$ is not influenced by free volume in contrast with $\chi_{0},{ }^{23.24}$ and $\chi_{0} p_{1}=1 / 3$ is also satisfied.

\section{Determination of $p_{1}, p_{2}, \theta$, and $\psi$}

Kamide and Matsuda ${ }^{2}$ showed that the terms more than or equal to $v_{\mathrm{p}}{ }^{3}$ can be neglected to make the theoretical critical concentration $v_{\mathrm{p}}^{\mathrm{c}}($ theo) coincident with the experimental value $v_{\mathrm{p}}^{\mathrm{c}}(\exp )$. First calculate $\chi_{0}^{\mathrm{c}}$ and $v_{\mathrm{p}}^{\mathrm{c}}$ (theo) by solving eq 7 and 8 with assumed values of $p_{1}$ and $p_{2}$. Evaluate the square average of the difference between $v_{\mathrm{p}}^{\mathrm{c}}$ (theo) and $v_{\mathrm{p}}^{\mathrm{c}}(\exp ), \delta$, defined by

$$
\begin{aligned}
\delta=\sum_{i=1}^{N}\left(v_{\mathrm{p}}^{\mathrm{c}}(\exp )-v_{\mathrm{p}}^{\mathrm{c}}(\text { theo })\right)_{i}{ }^{2} / N_{0} \\
\\
\left(N_{0}, \text { total number of samples }\right)
\end{aligned}
$$

for a given combination of $p_{1}$ and $p_{2}$. Finally determine the most probable $p_{1}$ and $p_{2}$ as a pair which gives minimum $\delta$. Substituting $\chi_{0}^{\mathrm{c}}$, calculated for the most probable $p_{1}$ and $p_{2}$, and the experimental critical temperature $T_{\mathrm{c}}$ (exp) into eq 16, and plotting the relation of $1 / T_{\mathrm{c}}(\exp ) v s . \chi_{0}^{\mathrm{c}}($ theo), we obtain $\theta$ and $\psi$.

Koningsveld et al. ${ }^{1,9}$ defined the polymersolvent interaction parameter $g$ by eq 24 ,

$$
g=\sum_{i=0}^{n} g_{i} v_{\mathrm{p}}^{i}
$$

where $g_{i}$ is the concentration dependent parameter. Equation 8 can be rearranged using eq 24 into

$$
\begin{aligned}
Y & \equiv g_{1}-g_{2}+4 g_{2} v_{\mathrm{p}}^{\mathrm{c}} \\
& =\frac{1}{6}\left[\frac{1}{\left(1+v_{\mathrm{p}}^{\mathrm{c}}\right)^{2}}-\frac{X_{z}}{\left(X_{w} v_{\mathrm{p}}^{c}\right)^{2}}\right]
\end{aligned}
$$

for the case of $n=2$. Substituting $X_{w}$ and $v_{\mathrm{p}}^{\mathrm{c}}(\exp )$ into eq 25 we obtain the relation of $T$ and $v_{\mathrm{p}}^{\mathrm{c}}$, from which $g_{1}$ and $g_{2}$ can be determined using curve fitting method. $g_{0}$ depends on the temperature through the relation,

$$
\begin{aligned}
g_{0} \equiv & g_{00}+g_{01} / T_{\mathbf{c}} \\
= & \frac{1}{2}\left[\frac{1}{1-v_{\mathrm{p}}^{c}}+\frac{1}{X_{w} v_{\mathrm{p}}^{c}}\right. \\
& \left.+2 g_{1}\left(1-3 v_{\mathrm{p}}^{c}\right)+6 g_{2}\left(1-2 v_{\mathrm{p}}^{c}\right) v_{\mathrm{p}}^{c}\right]
\end{aligned}
$$

Substitution of $X_{w}, X_{z}, T_{\mathrm{c}}(\exp )$ data into eq 26 enables us to estimate $g_{00}$ and $g_{01}$.

The following relation holds between $\chi$ and $g$

$$
\chi=g-\left(1-v_{\mathrm{p}}\right) \frac{\partial g}{\partial v_{\mathrm{p}}}
$$

and the parameters $\chi_{0}, p_{1}$, and $p_{2}$ in eq 1 can be expressed in terms of $g_{00}, g_{01}, f_{1}$ and $g_{2}$ as follows:

$$
\begin{aligned}
& \chi_{0}=g_{00}-g_{1}+\frac{g_{01}}{T} \\
& p_{1}=2 \frac{g_{1}-g_{2}}{g_{00}-g_{1}+g_{01} / T} \\
& p_{2}=3 \frac{g_{2}}{g_{00}-g_{1}+g_{01} / T}
\end{aligned}
$$

Comparing eq 16 with eq 28 , we obtain

$$
\begin{aligned}
& \psi=\frac{1}{2}-g_{00}+g_{1} \\
& \theta=g_{01} / \psi
\end{aligned}
$$

\section{APPLICATION TO EXPERIMENTAL DATA}

The literature data on the critical solution 
points $\left(v_{\mathrm{p}}^{\mathrm{c}}(\exp )\right.$ and $\left.T_{\mathrm{c}}(\exp )\right)$ on polystyrene (PS), ${ }^{1,10-16}$ and polyethylene(PE) $)^{17-22}$ in single solvents were analyzed. It should be remembered that except for dimethoxy methane by Siow et al. $^{15}$ and cyclohexane (in part) by Koningsveld and his coworkers, ${ }^{1}$ all the data on PS solutions were made by Kaneko, Kuwahara, and their collaborators at Sapporo, ${ }^{10-14,16}$ who used PS samples with $X_{w} / X_{n}<1.10$ (in particular for samples with the weight-average molecular weight $M_{w} \times 10^{-4}=3: 7 \quad$ to $\left.40, \quad X_{w} / X_{n}<1.06\right)$ manufactured by Pressure Chemical Co. (Pittsburg, USA) and the majority of literature data on PE solutions was cited from works by Nakajima, Hamada et al. at Kyoto, ${ }^{19,20}$ who employed PE fractions isolated by successive precipitation fractionation or solid extraction (column) method from whole polymers.

Here, in the case where $v_{\mathrm{p}}^{\mathrm{c}}(\exp )$ and $T_{\mathrm{c}}(\exp )$ are described in the literature, we employed these data without recalculation and in the case where neither $v_{\mathrm{p}}^{\mathrm{c}}(\exp )$ nor $T_{\mathrm{c}}(\exp )$ are given in the literature, we regarded the threshold cloud point ( $v_{\mathrm{p}}^{\text {tep }}$ and $T_{\text {tcp }}$ ) as the critical point and if no threshold cloud point data are available, we estimated from the cloud point curve in the literature. For the polymer with the large molecular weight and sharp distribution, the difference between $v_{\mathrm{p}}^{\mathrm{c}}(\exp )$ and $v_{\mathrm{p}}^{\mathrm{tcp}}(\exp )$ can become small enough to be neglected. ${ }^{3}$ However, for the polymer with small molecular weight and broad distribution, special attention should be paid, because the difference becomes of the order of 0.01 . In addition, the temperature dependence of specific volumes of the polymer and the solvent should be carefully taken into account to convert the cloud point curve, usually expressed in terms of the weight fraction. Of course, $p_{1}, p_{2}, \theta$, and $\psi$ determined by KKS and KM method are significantly affected by the accuracy of $g_{\mathbf{p}}^{\mathfrak{c}}(\exp )$ and $T_{\mathrm{c}}(\exp )$.

Tables I and II show data for the polymer-solvent systems used for analysis, the number of samples for each system and the method, by which the critical solution data are estimated. In these tables, $R$ denotes the case when $v_{\mathrm{p}}^{\mathrm{c}}(\exp )$ is determined as $v_{\mathrm{p}}$ of the two phase volume ratio $R=1$, diameter and tcp denotes the case when $v_{\mathrm{p}}^{\mathrm{c}}(\exp )$ is determined from diameter and the threshold cloud point curve, respectively. These tables contain also the correlation coefficients $r$ of $1 / T_{\mathrm{c}} v s . \chi_{0}^{\mathrm{c}}$ (eq 16) or $Y v s$. $v_{\mathrm{p}}^{\mathrm{c}}\left(\right.$ eq 25) or $g_{0} v s .1 / T_{\mathrm{c}}$ (eq 26). Obviously accuracy of $v_{\mathrm{p}}^{\mathrm{c}}(\exp )$ is lower than that of $T_{c}(\exp )$ for any polymer-solvent systems.

\section{RESULTS AND DISCUSSION}

Tables III and IV summarized $p_{1}, p_{2}, \theta$ and $\psi$ values for PS- and PE-solvent systems, evaluated by KM, KKS, and SF methods. The values in parenthesis are cited from literature.

\section{Polystyrene}

For PS/methyl ethyl ketone(LCSP), PS/toluene(LCSP), PS/isopropyl acetate(UCSP), PS $n$-propyl acetate(UCSP), and PS/dimethoxy methane(LCSP), the correlation coefficient $r$ between $Y$ and $v_{\mathrm{p}}^{\mathrm{c}}$ is low $(<0.55)$ and in consequence, the reliability of $p_{1}$ and $p_{2}$ obtained by KKS method is not high for these systems (Table I). In contrast to this, $r$ between $1 / T_{\mathrm{c}}$ and $\chi_{0}^{\mathrm{c}}$ (by KM method) is without exception larger than 0.98. Except for PS/toluene(LCSP), PS/benzene(LCSP), PS/ isopropyl acetate(LCSP), PS/n-propyl acetate (LCSP), the $p_{1}$ value determined for PS solutions by KM method can be almost regarded as constant $(0.638 \pm 0.035)$. This value is near to the theoretically expected value $(2 / 3)$. The range of variation $( \pm 0.035)$ in $p_{1}$ may contain the solvent effect together with the experimental uncertainty. Except for PS/cyclopentane, the $p_{2}$ value for LCSP is always negative, lying between -2.0 and -0.202 and, except PS/isopropyl acetate and $n$-propyl acetate systems, in which $p_{2}$ is slightly nega- 
Table I. Method for determining the critical point, number of samples and correlation coefficient $r$ of Shultz-Flory Koningsveld et al. and Kamide-Matsuda plots for polystyrene-solvent systems

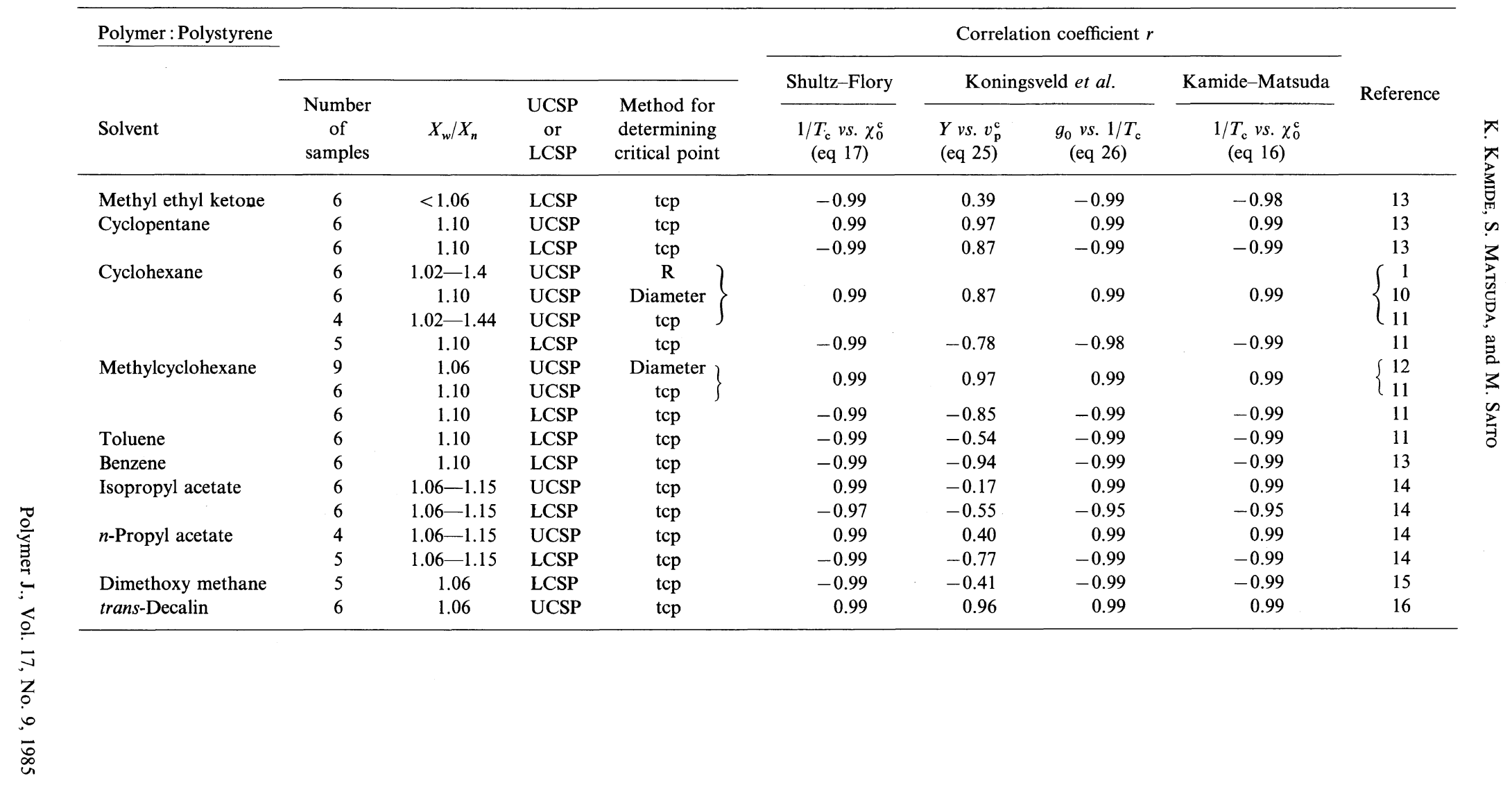


Table II. Method for determining the critical point, number of samples and correlation coefficient $r$ of Shultz-Flory Koningsveld $e t$ al. and Kamide-Matsuda plots for polyethyrene-solvent systems

\begin{tabular}{|c|c|c|c|c|c|c|c|c|c|}
\hline \multirow{3}{*}{$\begin{array}{l}\text { Polymer: Polyethyren } \\
\text { Solvent }\end{array}$} & \multirow{3}{*}{$\begin{array}{l}\text { Number } \\
\text { of } \\
\text { samples }\end{array}$} & \multirow[b]{3}{*}{$X_{w} / X_{n}$} & \multirow{3}{*}{$\begin{array}{l}\text { UCSP } \\
\text { or } \\
\text { LCSP }\end{array}$} & \multirow{3}{*}{$\begin{array}{l}\text { Method for } \\
\text { determining } \\
\text { critical point }\end{array}$} & \multicolumn{4}{|c|}{ Correlation coefficient $r$} & \multirow{3}{*}{ Reference } \\
\hline & & & & & \multirow{2}{*}{$\frac{\text { Shultz-Flory }}{\begin{array}{c}1 / T_{\mathrm{c}} v s . \chi_{0}^{\mathrm{c}} \\
(\text { eq } 17)\end{array}}$} & \multicolumn{2}{|c|}{ Koningsveld et al. } & \multirow{2}{*}{ 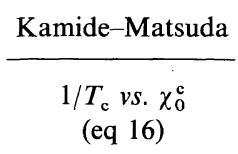 } & \\
\hline & & & & & & $\begin{array}{l}Y v s . v_{\mathrm{p}}^{\mathrm{c}} \\
(\mathrm{eq} 25)\end{array}$ & $\begin{array}{c}g_{0} \text { vs. } 1 / T_{\mathrm{c}} \\
\quad(\text { eq } 26)\end{array}$ & & \\
\hline \multirow[t]{2}{*}{$n$-Butyl acetate } & 4 & $\mathrm{~F}^{\mathrm{c}}$ & UCSP & tcp & 0.98 & -0.91 & 0.99 & -0.99 & 18 \\
\hline & 4 & $\mathrm{~F}^{\mathrm{c}}$ & LCSP & tcp & -0.97 & -0.86 & -0.97 & -0.97 & 18 \\
\hline$n$-Pentane & 3 & $\mathrm{~F}^{\mathrm{b}}$ & LCSP & tcp & -0.99 & -0.98 & -0.99 & -0.99 & 19 \\
\hline$n$-Hexane & 4 & $\mathrm{~F}^{\mathrm{b}}$ & LCSP & tcp & -0.99 & -0.93 & -0.99 & -0.99 & 19 \\
\hline$n$-Heptane & 4 & $\mathrm{~F}^{\mathrm{b}}$ & LCSP & tcp & -0.99 & 0.89 & -0.99 & -0.98 & 19 \\
\hline$n$-Octane & 4 & $\mathrm{~F}^{\mathbf{b}}$ & LCSP & tcp & -0.99 & 0.96 & -0.97 & -0.98 & 19 \\
\hline \multirow[t]{3}{*}{$n$-Octyl alcohol } & 4 & $\mathrm{~W} \& \mathrm{~F}$ & UCSP & tep & 0.99 & -0.87 & 0.99 & 0.99 & 21 \\
\hline & 4 & $\mathrm{~W} \& \mathrm{~F}$ & LCSP & tcp & -0.98 & -0.86 & -0.99 & -0.98 & 21 \\
\hline & 4 & $F^{a}$ & UCSP & tcp & 0.99 & -0.93 & 0.99 & 0.99 & 20 \\
\hline$n$-Decyl alcohol & 3 & $\mathrm{~F}^{\mathrm{a}}$ & UCSP & tcp & 0.99 & 0.79 & 0.99 & 0.99 & 20 \\
\hline$n$-Lauryl alcohol & 3 & $\mathrm{~F}^{\mathrm{a}}$ & UCSP & tcp & 0.99 & -0.98 & 0.99 & 0.99 & 20 \\
\hline p-tert-Amyl phenol & 4 & $F^{a}$ & UCSP & tcp & 0.99 & -0.49 & 0.99 & 0.99 & 20 \\
\hline$p$-Octyl phenol & 4 & $\mathrm{~F}^{\mathrm{a}}$ & UCSP & tcp & 0.99 & 0.09 & 0.99 & 0.99 & 20 \\
\hline$p$-Nonyl phenol & 3 & $\mathrm{~F}^{\mathrm{a}}$ & UCSP & tcp & 0.99 & -0.30 & 0.99 & 0.99 & 20 \\
\hline Anisole & 3 & $\mathrm{~F}^{\mathrm{a}}$ & UCSP & tcp & 0.99 & -0.99 & 0.99 & 0.99 & 20 \\
\hline Benzyl phenyl ether & 3 & $\mathrm{~F}^{\mathrm{a}}$ & UCSP & tcp & 0.97 & -0.30 & 0.99 & 0.98 & 20 \\
\hline Diphenyl & 4 & $F^{b}$ & UCSP & tcp & 0.96 & 0.96 & 0.99 & 0.99 & 22 \\
\hline Diphenyl methane & 6 & $F^{b}$ & UCSP & tcp & 0.97 & 0.72 & 0.99 & 0.99 & 22 \\
\hline \multirow[t]{2}{*}{ Diphenyl ether } & 3 & W \& F & UCSP & $\mathrm{R}$ & 0.98 & -0.47 & 0.99 & 0.99 & 17 \\
\hline & 6 & $F^{b}$ & UCSP & tcp & 0.99 & 0.07 & 0.99 & 0.99 & 22 \\
\hline
\end{tabular}

W, Whole polymer; F, Fractionated polymer $\left(\mathrm{F}^{\mathrm{a}}\right.$, by SPF; $\mathrm{F}^{\mathrm{b}}$, by column fractionation (solid extraction); $\mathrm{F}^{\mathrm{c}}$, by $\mathrm{SSF}$ ) 
Table III. Concentration dependence of $\chi$-parameter $p_{1}, p_{2}$, Flory temperature $\theta$, and entropy parameter $\psi$ of the critical point for polystyrene-solvent systems

\begin{tabular}{|c|c|c|c|c|c|c|c|c|c|c|c|}
\hline \multirow{3}{*}{$\begin{array}{l}\text { Polymer : Polystyrene } \\
\text { Solvent }\end{array}$} & \multirow{3}{*}{$\begin{array}{l}\text { UCSP } \\
\text { or } \\
\text { LCSP }\end{array}$} & \multicolumn{10}{|c|}{ Methods } \\
\hline & & \multicolumn{4}{|c|}{ Kamide--Matsuda } & \multicolumn{4}{|c|}{ Koningsveld et al. } & \multicolumn{2}{|c|}{ Shultz-Flory } \\
\hline & & $p_{1}$ & $p_{2}$ & $\theta$ & $\psi$ & $p_{1}$ & $p_{2}$ & $\theta$ & $\psi$ & $\theta(\theta$ ref $)$ & $\psi(\psi$ ref $)$ \\
\hline Methyl ethyl ketone & LCSP & 0.618 & -0.208 & 423.6 & -0.44 & 0.550 & -0.262 & 423.8 & -0.31 & $423.1\left(422^{* a}\right)$ & $-0.63\left(-0.529^{* a}\right)$ \\
\hline \multirow{2}{*}{ Cyclopentane } & UCSP & 0.615 & 0.404 & 292.1 & 0.16 & 0.606 & 0.497 & 292.1 & 0.18 & $292.7\left(293^{* a}\right)$ & $0.53\left(0.548^{* a}\right)$ \\
\hline & LCSP & 0.631 & 0.331 & 428.5 & -0.25 & 0.611 & 0.468 & 428 & -0.27 & $427.4\left(427^{* a}\right)$ & $-0.81\left(-0.858^{* a}\right)$ \\
\hline \multirow[t]{4}{*}{ Cyclohexane } & UCSP & $(0.642$ & 0.190 & 305.1 & $0.27^{* \mathrm{e}}$ ) & $(0.623$ & 0.308 & 305.2 & $0.29 * \mathrm{e})$ & $306.5\left(306.2^{* b}\right)$ & $0.75 \quad\left(0.78^{* b}\right)$ \\
\hline & & - & - & - & - & $(0.623$ & 0.290 & 306.4 & $0.30^{* \mathrm{c}}$ ) & $-\left(307.0^{* d}\right)$ & - $\left(0.79^{* d}\right)$ \\
\hline & LCSP & 0.638 & -0.498 & 488.6 & -0.58 & 0.621 & -0.305 & 488.3 & -0.60 & $486.8\left(486.0^{* d}\right)$ & $-1.21\left(-1.19^{* \mathrm{~d}}\right)$ \\
\hline & & $(0.602$ & 0.347 & 487.2 & $\left.-0.42^{* e}\right)$ & $(0.571$ & -0.047 & 487.5 & $\left.-0.61^{* \mathrm{e}}\right)$ & $-\left(486.0^{* \mathrm{e}}\right)$ & $-\left(-1.20^{* \mathrm{e}}\right)$ \\
\hline \multirow[t]{2}{*}{ Methyl cyclohexane } & UCSP & $(0.602$ & 0.234 & 340.2 & $0.25^{* \mathrm{e}}$ ) & $(0.602$ & 0.363 & 339.6 & $0.27^{* \mathrm{e}}$ ) & $342.3\left(344^{* d}\right)$ & $0.61 \quad\left(0.56^{* d}\right)$ \\
\hline & LCSP & $(0.649$ & -1.183 & 487.9 & $\left.-0.54^{* \mathrm{e}}\right)$ & $(0.643$ & -1.008 & 487.8 & $\left.-0.56^{* \mathrm{e}}\right)$ & $485.1\left(484^{* d}\right)$ & $-0.96\left(-0.94^{* \mathrm{~d}}\right)$ \\
\hline Toluene & LCSP & 0.494 & -0.922 & 550.4 & $-1: 36$ & 0.501 & -0.475 & 550.3 & -1.20 & $549.8\left(550^{* d}\right)$ & $-2.02\left(-1.92^{* \mathrm{~d}}\right)$ \\
\hline Benzene & LCSP & 0.388 & -1.781 & 524.3 & -1.81 & 0.382 & -1.655 & 524.2 & -1.82 & $523.7\left(523^{* a}\right)$ & $-2.19\left(-1.79^{* a}\right)$ \\
\hline \multirow[t]{2}{*}{ Isopropyl acetate } & UCSP & 0.673 & -0.034 & 240.8 & 0.11 & 0.673 & -0.082 & 240.6 & 0.13 & $245.3\left(246^{* f}\right)$ & $0.29 \quad(0.32 * \mathrm{f})$ \\
\hline & LCSP & 0.839 & -2.000 & 398.1 & -0.46 & 0.773 & -1.594 & 394.6 & -0.46 & $389.5\left(380^{* f}\right)$ & $-0.71\left(-0.46^{* f}\right)$ \\
\hline \multirow{2}{*}{$n$-Propyl acetate } & UCSP & 0.643 & -0.018 & 192.3 & 0.21 & 0.623 & 0.168 & 192.7 & 0.22 & $193.2\left(193^{* f}\right)$ & $0.60 \quad\left(0.63^{* \mathrm{f}}\right)$ \\
\hline & LCSP & 0.797 & -1.440 & 456.3 & -0.47 & 0.769 & -1.204 & 455.6 & -0.49 & $451.1\left(451^{* f}\right)$ & $-0.96\left(-0.85^{* f}\right)$ \\
\hline \multirow{2}{*}{$\begin{array}{l}\text { Dimethoxy methane } \\
\text { trans-Decalin }\end{array}$} & LCSP & 0.650 & -0.202 & 389.1 & -0.25 & 0.642 & -0.083 & 388.0 & -0.24 & $386.0 \quad(-)$ & $-0.61 \quad(-)$ \\
\hline & UCSP & 0.630 & 0.240 & 292.7 & 0.33 & 0.623 & 0.338 & 292.5 & 0.36 & $293.7(-)$ & 0.95 \\
\hline
\end{tabular}

${ }^{\mathrm{a}}$ ref $13 .{ }^{\mathrm{b}}$ ref $3 .^{\mathrm{c}}$ ref $2 .^{\mathrm{d}}$ ref $4 .^{\mathrm{e}}$ ref $1 .^{\mathrm{f}}$ ref 14. 
Table IV. Concentration dependence of $\chi$-parameter $p_{1}, p_{2}$, Flory temperature $\theta$, and entropy parameter $\psi$ of the critical point for polyethylene-solvent systems

\begin{tabular}{|c|c|c|c|c|c|c|c|c|c|c|c|c|}
\hline \multirow{4}{*}{$\begin{array}{l}\text { Polymer : Polyethylene } \\
\text { Solvent } \\
n \text {-Butyl acetate }\end{array}$} & \multirow{4}{*}{$\begin{array}{c}\begin{array}{c}\text { UCSP } \\
\text { or } \\
\text { LCSP }\end{array} \\
\text { UCSP }\end{array}$} & \multicolumn{11}{|c|}{ Methods } \\
\hline & & \multicolumn{4}{|c|}{ Kamide-Matsuda } & \multicolumn{4}{|c|}{ Koningsveld et al. } & \multicolumn{3}{|c|}{ Shultz-Flory } \\
\hline & & \multirow{2}{*}{$\begin{array}{l}p_{1} \\
2.98\end{array}$} & \multirow{2}{*}{$\frac{p_{2}}{-34.6}$} & \multirow{2}{*}{$\frac{\theta}{453.8}$} & \multirow{2}{*}{$\frac{\psi}{0.89}$} & \multirow{2}{*}{$\frac{p_{1}}{3.100}$} & \multirow{2}{*}{$\frac{p_{2}}{-36.6}$} & \multirow{2}{*}{$\frac{\theta}{453.8}$} & \multirow{2}{*}{$\frac{\psi}{0.93}$} & \multirow{2}{*}{$\frac{\theta(\theta \mathrm{ref})}{482.1\left(483^{* a}\right)}$} & \multicolumn{2}{|c|}{$\psi(\psi$ ref $)$} \\
\hline & & & & & & & & & & & 0.61 & $\left(0.65^{* a}\right)$ \\
\hline & LCSP & 4.63 & -38.0 & 506.5 & -1.15 & 3.742 & -31.59 & 499.9 & -1.27 & $474.9\left(471^{* a}\right)$ & -1.17( & $\left.-1.12^{* a}\right)$ \\
\hline$n$-Pentane & LCSP & 1.95 & -23.4 & 366.7 & -1.82 & 4.529 & -42.69 & 376.2 & -1.78 & $351.8\left(353^{* b}\right)$ & -1.06( & $\left.-1.3^{* b}\right)$ \\
\hline$n$-Hexane & LCSP & 0.662 & -6.53 & 410.4 & -0.93 & 0.675 & -6.54 & 410.3 & -0.95 & $407.3\left(406.3^{* \mathrm{~b}}\right)$ & $-0.98 i$ & $\left.-1.0^{* b}\right)$ \\
\hline$n$-Heptane & LCSP & -1.5 & 12.9 & 446.5 & -1.89 & -3.76 & 34.60 & 446.5 & -2.68 & $447.1\left(446.9^{* \mathrm{~b}}\right)$ & -1.16( & $\left.-1.2^{* b}\right)$ \\
\hline$n$-Octane & LCSP & -1.0 & 7.7 & 482.9 & -1.64 & -2.873 & 26.12 & 481.6 & -2.14 & $484.4\left(483.0^{* \mathrm{~b}}\right)$ & -1.22( & $\left.-1.1^{* \mathrm{~b}}\right)$ \\
\hline \multirow[t]{3}{*}{$n$-Octyl alcohol } & UCSP & 1.7 & -33.7 & 440.9 & 1.53 & 3.435 & -59.0 & 436.7 & 1.52 & $445.0\left(444^{* c}\right)$ & 1.21 & $\left(1.16^{* c}\right)$ \\
\hline & LCSP & 1.8 & -37.0 & 621.9 & -7.40 & 2.712 & -51.43 & 622.4 & -7.13 & $620.6\left(621^{* c}\right)$ & -5.76( & $\left.-5.4^{* c}\right)$ \\
\hline & UCSP & 0.48 & -25.6 & 446.3 & 2.59 & 1.440 & -43.79 & 445.8 & 2.47 & $452.9\left(453.1^{* \mathrm{~d}}\right)$ & 1.15 & $\left(1.15^{* d}\right)$ \\
\hline n-Decyl alcohol & UCSP & -1.82 & 6.02 & 425.7 & 2.82 & -2.688 & 11.24 & 427.6 & 2.65 & $425.5\left(426.3^{* d}\right)$ & 1.47 & $\left(1.44^{* d}\right)$ \\
\hline$n$-Lauryl alcohol & UCSP & -0.304 & -3.40 & 409.0 & 2.47 & -0.249 & -5.036 & 409.2 & 2.39 & $410.1\left(410.3^{* d}\right)$ & 1.67 & $\left(1.64^{* d}\right)$ \\
\hline p-tert-Amyl phenol & UCSP & -2.13 & 2.30 & 470.2 & 2.54 & -1.923 & -6.158 & 472.0 & 2.17 & $472.1\left(472.2^{* d}\right)$ & 1.07 & $\left(1.07^{* d}\right)$ \\
\hline$p$-Octyl phenol & UCSP & -1.52 & -0.912 & 446.1 & 3.26 & -2.744 & 0.997 & 450.6 & 2.89 & $448.2\left(447.5^{* d}\right)$ & 1.47 & $\left(1.48^{* d}\right)$ \\
\hline$p$-Nonyl phenol & UCSP & 0.175 & -2.71 & 434.1 & 1.78 & -0.095 & -1.277 & 435.7 & 1.77 & $435.8\left(435.4^{* d}\right)$ & 1.55 & $\left(1.62^{* d}\right)$ \\
\hline Anisole & UCSP & 1.15 & -39.1 & 422.6 & 2.61 & 1.655 & -49.63 & 422.1 & 2.59 & $426.3\left(426.5^{* d}\right)$ & 1.45 & $\left(1.41^{* d}\right)$ \\
\hline Benzyl phenyl ether & UCSP & -0.823 & -20.1 & 461.6 & 2.95 & -1.945 & -12.17 & 464.2 & 2.74 & $464.0\left(464.5^{* \mathrm{~d}}\right)$ & 1.32 & $\left(1.38^{* d}\right)$ \\
\hline Diphenyl & UCSP & -1.32 & 9.32 & 400.5 & 1.84 & -2.064 & 15.86 & 401.1 & 1.95 & $399.8\left(400.5^{* e}\right)$ & 1.19 & $\left(1.17^{* e}\right)$ \\
\hline Diphenyl methane & UCSP & -0.89 & 3.1 & 414.5 & 1.61 & -1.970 & 10.66 & 418.6 & 1.58 & $413.7\left(415.2^{* e}\right)$ & 1.13 & $\left(1.06^{* e}\right)$ \\
\hline \multirow[t]{2}{*}{ Diphenyl ether } & UCSP & 0.611 & -2.483 & 427.5 & 1.04 & 0.400 & -1.50 & 431.4 & 0.98 & $431.9(-)$ & 0.96 & $(-)$ \\
\hline & UCSP & -0.31 & -1.6 & 433.0 & 1.53 & -0.698 & 0.471 & 435.1 & 1.49 & $434.1\left(436.9^{* e}\right)$ & 1.12 & $\left(1.00^{* e}\right)$ \\
\hline
\end{tabular}

${ }^{a}$ ref $18 .{ }^{b}$ ref $19 .{ }^{\mathrm{c}}$ ref $21 .{ }^{\mathrm{d}}$ ref $20 .{ }^{\mathrm{e}}$ ref 22 


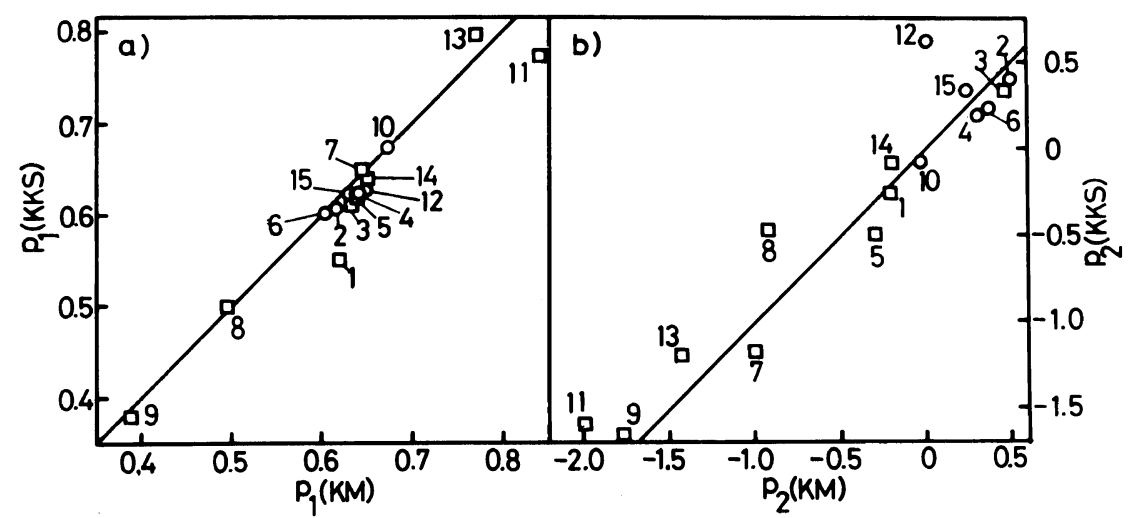

Figure 1. Correlations between $p_{1}(\mathrm{KKS})$ and $p_{1}(\mathrm{KM})$ and correlations between $p_{2}(\mathrm{KKS})$ and $p_{2}(\mathrm{KM})$, both for polystyrene solutions: rectangle, LCSP; circle, UCSP: 1 , methyl ethyl ketone (LCSP) ${ }^{13} ; 2$, cyclopentane (UCSP) $)^{13} ; 3$, cyclopentane $(\mathrm{LCSP})^{13} ; 4$, cyclohexane $(\mathrm{UCSP})^{1,10,11} ; 5$, cyclohexane $(\mathrm{LCSP})^{11}$; 6 , methylcyclohexane (UCSP) $)^{11,12} ; 7$, methylcyclohexane $(\mathrm{LCSP})^{11} ; 8$, toluene (LCSP) ${ }^{11} ; 9$, benzene $(\text { LCSP) })^{13} ; 10$, isopropyl acetate (UCSP) $)^{14} ; 11$, isopropyl acetate (UCSP) ${ }^{14} ; 11$, isopropyl acetate (LCSP) ${ }^{14}$; $12, n$-propyl acetate (UCSP) ${ }^{14} ; 13, n$-propyl acetate $(\operatorname{LCSP})^{14} ; 14$, dimethoxy methane (LCSP) ${ }^{15} ; 15$, transdecalin (UCSP). ${ }^{16}$

tive $(-0.03$ and -0.02$)$, the $p_{2}$ value for UCSP is positive $(0.297 \pm 0.107)$. This mean value is slightly smaller than the theoretical value $(1 / 2)$.

Figure la) shows the plot of $p_{1}$, evaluated by KKS method $\left(p_{1}(\mathrm{KKS})\right)$ versus $p_{1}$, evaluated by $\mathrm{KM}$ method $\left(p_{1}(\mathrm{KM})\right)$ and Figure $\left.1 \mathrm{~b}\right)$ is similar plot of $p_{2}$. KM and $\mathrm{KKS}$ methods give almost the same values of $p_{1}$ and $p_{2}$ (hereafter referred to as $p_{1}(\mathrm{KM}), p_{1}(\mathrm{KKS}), p_{2}(\mathrm{KM})$, and $p_{2}(\mathrm{KKS})$ ).

$$
\begin{gathered}
p_{1}(\mathrm{KKS})=0.889 p_{1}(\mathrm{KM})+0.052, \\
r=0.9813 \\
p_{2}(\mathrm{KKS})=0.927 p_{2}(\mathrm{KM})+0.150, \\
r=0.9714
\end{gathered}
$$

The Flory temperature, obtained by three methods, is practically independent of the methods employed. $\psi$ is positive for UCSP and negative for LCSP as expected, irrespective of the methods used for evaluation. These are observed not only for PS, but also for PE, strongly suggesting that $\psi$ cannot be regarded as temperature-independent over wide range of temperature, which is assumed in KKS and KM methods and the polymer solution should be athermal at a temperature between UCSP and LCSP. Absolute values of $\psi,|\psi|$ increases in the order:

$$
|\psi(\mathrm{KM})| \cong|\psi(\mathrm{KKS})|<|\psi(\mathrm{SF})|
$$

As pointed out for $\mathrm{PS} / \mathrm{CH}$ and $\mathrm{PS} / \mathrm{MCH}$ systems in the previous paper, ${ }^{2}$ the fact that $|\psi(\mathrm{SF})|$ is larger than $|\psi(\mathrm{KM})|$ and $|\psi(\mathrm{KKS})|$ is mainly caused from the ignorance of the concentration dependence of $\chi$ parameter in SF method. Among three methods the following relations hold:

$$
\begin{array}{rr}
\psi(\mathrm{KKS})=0.983 \psi(\mathrm{KM})+0.017, & r=0.9963 \\
\psi(\mathrm{SF})=1.554 \psi(\mathrm{KM})+0.076, & r=0.9553 \\
\psi(\mathrm{SF})=1.578 \psi(\mathrm{KKS})+0.094, & r=0.9571
\end{array}
$$

For PS/cyclopentane (CP), /CH, and / $\mathrm{MCH}$ systems, $p_{1}$ values for LCSP or UCSP are very similar to each other, but difference in $\psi$ values between $\mathrm{CP}$ and $\mathrm{CH}$ is much larger than that between $\mathrm{CH}$ and $\mathrm{MCH}$ for both CSP. In other words, the skeleton structure of the solvent (i.e., five- or six-membered ring structure) is a more important factor than the substituent group to the skeleton, covering the thermody- 
namic interaction between the polymer and solvent.

Even if the solvents have similar molecular weights and almost the same molecular shapes, $p_{1}$ and also $p_{2}$ differ remarkably depending on whether the solvent is aliphatic or aromatic. For $\mathrm{PS} / \mathrm{CH}$ and $\mathrm{PS} / \mathrm{MCH}$ systems, $p_{1}$ for LCSP was found to be $0.64-0.65$ by $\mathrm{KM}$ method. In contrast to this, for PS/benzene and PS/toluene systems $p_{1}$ for LCSP was 0.39 and 0.49 by KM method, respectively. An aromatic solvent acts as a better solvent against PS.

Figure 2 shows the plot of $p_{2}$ against $p_{1}$, both estimated by KM method, for PS solutions. Here, unfilled circle and rectangle correspond to UCSP and LCSP, respectively. The point theoretically expected when $A_{2}=A_{3}=$ $A_{4}=0$ at $\theta$ temperature, is denoted as a filled circle. It is obvious that the experimental points for UCSP are not far from the theoretical point. On the other hand, the data points scatter for LCSP, showing negative $p_{2}$.

Figure 3 shows ${ }^{1} \mathrm{H}$ NMR spectra of PS (the weight-average molecular weight $M_{w}=$ $23.2 \times 10^{4}$ by the light scattering method in benzene at $25^{\circ} \mathrm{C}^{3}$ ) solutions in hexadeutro benzene (benzene- $d_{6}$ ) and in $\mathrm{CH}$. In the former system, the spectra consists of five peaks, as denoted in the figure by a-e. Peaks a and $b$ are due to the proton attached to benzene ring, in racemic and meso configurations, of PS, respectively. Here, the intensity ratio of $a$ to $b$ was 2.28. Peak c is attributed to methin proton in the PS main chain. The NMR peak of the methylene proton splits into two peaks $\mathrm{d}$ and $\mathrm{e}$, corresponding to their racemic and meso configurations, respectively. In the meso configuration (peak b), the direction of the magnetic field induced by a circular current of benzene ring is exactly anti-parallel to that of the external field and then the magnetic shielding effect is weekened, resulting in a significant shift of the peak to higher than that in racemic configuration. The peaks $\mathrm{c}, \mathrm{d}$, and e, observed in higher magnetic field $(<3 \mathrm{ppm})$ for $\mathrm{PS} / \mathrm{CH}$

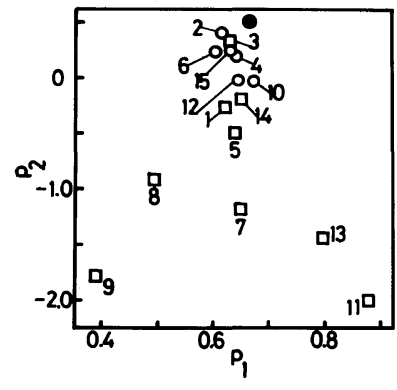

Figure 2. Plot of $p_{1}(\mathrm{MK})$ versus $p_{2}(\mathrm{KM})$ for polystyrene solutions: rectangle, LCSP; unfilled circle, UCSP; filled circle, the point theoretically expected when $A_{2}=$ $A_{3}=A_{4}=0$ at $\theta$. Key number has the same meaning as those in Figure 1.

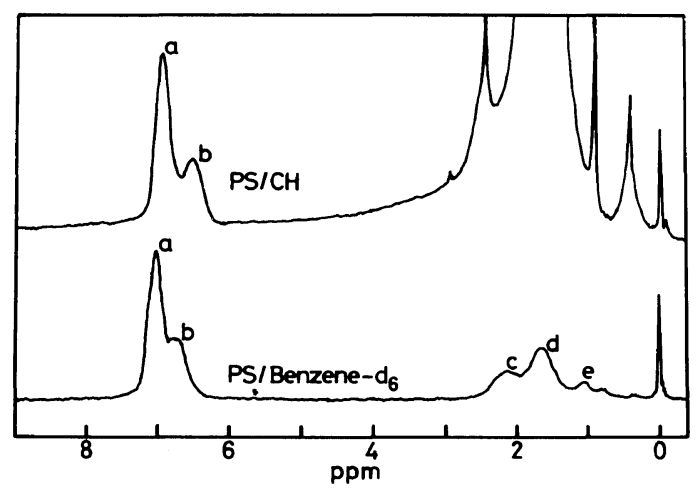

Figure 3. ${ }^{1} \mathrm{H}$ NMR spectra of polystyrene in benzene$d_{6}$ and in cyclohexane: for assignment of peaks a, b, c, d, and e see the text.

system, are overlapped by those of the solvent and only peaks a and $b$ are separately detected. Chemical shift of the proton attached to the benzene ring of PS in racemic configuration was observed at $7.04 \mathrm{ppm}$ in benzene and at $6.95 \mathrm{ppm}$ in $\mathrm{CH}$. Similar difference in the chemical shift for meso configuration $(0.21$ ppm) was observed between benzene and $\mathrm{CH}$. This kind of shift of peak a or b to lower magnetic field can be explained as follows: The magnetic shielding effect induced by circular electric current is strengthened by planar interaction between the benzene ring of PS and benzene as solvent. The interaction makes benzene a better solvent than $\mathrm{CH}$.

$p_{1}, p_{2}, \theta$, and $\psi$ values of UCSP for the 


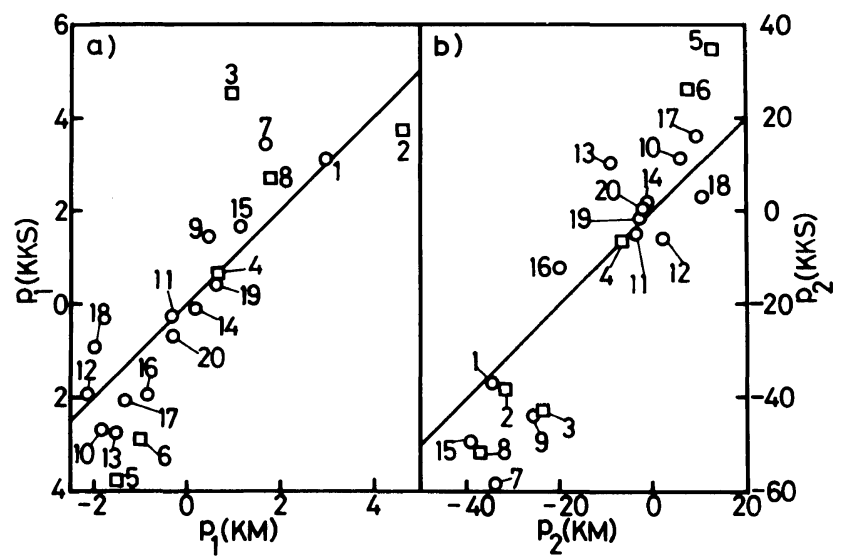

Figure 4. Correlations between $p_{1}(\mathrm{KKS})$ and $p_{1}(\mathrm{KM})$ and correlations between $p_{2}(\mathrm{KKS})$ and $p_{2}(\mathrm{KM})$, both for polystyrene solutions: rectangle, LCSP; circle, UCSP: $1, n$-butyl acetate (UCSP) ${ }^{18} ; 2, n$-butyl acetate $(\text { LCSP) })^{18} ; 3, n$-pentane $\left(\text { LCSP) }{ }^{19} ; 4, n \text {-hexane (LCSP) }{ }^{19} ; 5, n \text {-heptane (LCSP) }\right)^{19} ; 6, n$-octane $(\mathrm{LCSP})^{19} ; 7, n$-octyl alcohol $(\mathrm{UCSP})^{21} ; 8, n$-octyl alcohol $(\mathrm{LCSP})^{21} ; 9, n$-octyl alcohol $(\mathrm{UCSP})^{20} ; 10, n$ decyl alcohol $(\mathrm{UCSP})^{20} ; 11, n$-lauryl alcohol (UCSP) ${ }^{20} ; 12, p$-tert-amyl phenol (UCSP) ${ }^{20} ; 13, p$-octyl phenol (UCSP) ${ }^{20} ; 14, p$-nonyl phenol $(\mathrm{UCSP})^{20} ; 15$, anisole (UCSP) ${ }^{20} ; 16$, benzyl phenyl ether (UCSP) ${ }^{20}$; 17 , diphenyl (UCSP) ${ }^{22} ; 18$, diphenyl methane (UCSP) ${ }^{22} ; 19$, diphenylether (UCSP) ${ }^{17} ; 20$, diphenyl ether (UCSP). ${ }^{22}$

$\mathrm{PS} / \mathrm{CH}$ system were calculated from whole data on $v_{\mathrm{p}}^{\mathrm{c}}$ and $T_{\mathrm{c}}$, obtained by three different methods. The data of Kuwahara et al. ${ }^{10}$ by the diameter method gave $p_{1}=0.593, p_{2}=0.551$, $\theta=306.9$ and $\psi=0.22$, The threshold cloud point method ${ }^{11}$ gave $p_{1}=0.645, p_{2}=0.165$, $\theta=305.1$, and $\psi=0.27$. The phase volume ratio $\operatorname{method}^{1}$ yielding $p_{1}=0.631, p_{2}=0.221, \theta=$ 305.8 , and $\psi=0.27$. Obviously, from the latter two methods, we can obtain the same results, giving $p_{1}$ value similar with the theoretical value and also $\psi$ value, practically identical with the average value $(0.264)$ estimated from the second virial coefficient.

\section{Polyethylene}

For $\mathrm{PE} / p$-tert-amyl phenol(UCSP), $\mathrm{PE} / p$ octyl phenol(UCSP), PE/p-nonyl phenol (UCSP), PE/benzyl phenyl ether (UCSP), and PE/diphenyl ether(UCSP), the correlation between $Y$ and $v_{\mathrm{p}}^{\mathrm{c}}$ is low $(|r|<0.5)$. Even for these systems, $|r|$ between $1 / T_{\mathrm{c}}$ and $\chi_{0}^{\mathrm{c}}$ in $\mathrm{KM}$ method is larger than 0.97. $p_{1}$ and $p_{2}$, determined by $\mathrm{KM}$ method vary in the range $p_{1}=-1.82-2.98, \quad p_{2}=-39.1-9.32$ for
$\mathrm{UCSP}$, and $p_{1}=-1.5-4.63, p_{2}=-38.0$ 12.9 for LCSP. Obviously, the range of variation in $p_{1}$ and $p_{2}$ is much wider for $\mathrm{PE}$ than for PS, and only two system, PE $/ n$-hexane(LCSP) and PE/diphenyl ether(UCSP), have $p_{1}$ values in the vicinity of the theoretical value $(2 / 3)$.

Figure $4 a$ ) and b) show the relations between $p_{1}(\mathrm{KKS})$ and $p_{1}(\mathrm{KM})$ and between $p_{2}(\mathrm{KKS})$ and $p_{2}(\mathrm{KM})$, respectively. From the figure, we obtain

$$
\begin{aligned}
& p_{1}(\mathrm{KKS})=1.322 p_{1}(\mathrm{KM})-0.265 \\
& r=0.913
\end{aligned}
$$

and

$$
\begin{aligned}
& p_{2}(\mathrm{KKS})=1.436 p_{2}(\mathrm{KM})-0.398 \\
& r=0.942 \\
& \quad|\psi| \text { decreases in the order } \\
& \quad|\psi(\mathrm{KM})| \cong|\psi(\mathrm{KKS})|>|\psi(\mathrm{SF})|
\end{aligned}
$$

In PE solutions, $p_{2}$ often has an unexpectedly large negative value, which brings about larger $|\psi(\mathrm{KM})|$ than $|\psi(\mathrm{SF})|$. Therefore, if $\psi$ value, deduced from the temperature dependence of 
$A_{2}$ of PE solutions, $\psi\left(A_{2}\right)$ becomes available in the future, we can predict $\left|\psi\left(A_{2}\right)\right|>|\psi(\mathrm{SF})|$.

$$
\begin{aligned}
\psi(\mathrm{KKS})=0.987 \psi(\mathrm{KM})-0.114, & r=0.996 \\
\psi(\mathrm{SF})=0.680 \psi(\mathrm{KM})-0.196, & r=0.980 \\
\psi(\mathrm{SF})=0.684 \psi(\mathrm{KKS})-0.115, & r=0.977
\end{aligned}
$$

Among the literature data on $\mathrm{PE} /$ solvent systems, those by Koningsveld et al. ${ }^{17}$ for PE/ diphenylether are believed to have the highest accuracy, because the cloud point curves they constructed for three PE samples were constituted of 28,42 , and 48 data points and the critical points were determined by using the two phase volume ratio $R$, which gives directly the critical point, irrespective of the polymolecularity of the sample. It is wellknown that $\mathrm{PE}$ as polymerized whole polymers have extremely wide molecular weight distributions and PE fractions isolated by successive precipitation fractionation using proper solvent/non-solvent the system can never be regarded as monodisperse. Unfortunately, with exception of Koningsveld et al.'s experiments, ${ }^{17}$ no experiment was carried out to determine $v_{\mathrm{p}}^{\mathrm{c}}$ and $T_{\mathrm{c}}$ using $R$. The results, obtained by analyzing Nakajima et al.'s threshold cloud point curves for PE/diphenyl ether system are different remarkably from the data by Koningsveld et al. for the same polymer/solvent system. This means that the threshold cloud points, at least for PE fractions, do not coincide with the critical points with good accuracy and the wide variation of $p_{1}$ and $p_{2}$ is mainly due to low accuracy of the experiments.

By analyzing Koningsveld et al.'s data ${ }^{17}$ for the $\mathrm{PE} /$ diphenylether system, we obtain $p_{1}=$ 0.61 , using the KM method, which is close to the theoretical value. Koningsveld et al. ${ }^{17}$ concluded, only considering $g_{1}$, that the most reasonable supposition for $\mathrm{PE} /$ diphenyl ether system appears to be $g_{1}$ (accordingly, $\left.p_{1}\right)=0$, and $\delta$ (in eq 23), calculated assuming $p_{1}=p_{2}=0$ for the same data, is about $60 \%$ larger than that obtained using the values of $p_{1}$ and $p_{2}$, estimated by $\mathrm{KM}$ method for this system $\left(p_{1}=0.61\right.$ and $\left.p_{2}=-2.483\right)$. Similar analysis of Nakajima and his coworkers' data for the same system gave $p_{1}=-0.31$ by $\mathrm{KM}$ method. Note that the cloud point curves in Nakajima et al.'s study were constructed from only 4-8 (average 5) different concentration solutions. The large difference in $p_{1}$ indicates the experimental difficulty (and accordingly, the experimental uncertainty) contained in determination of the critical point of PE solutions, and as far as PE/diphenyl ether sys-

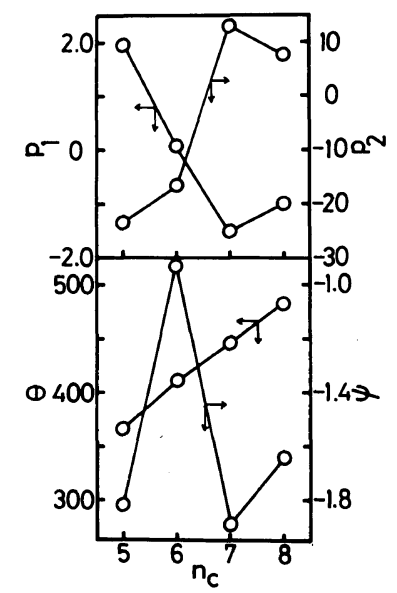

Figure 5. $p_{1}, p_{2}, \theta$, and $\psi$ plotted against the carbon number $n_{\mathrm{c}}$ constituting linear aliphatic hydrocarbons as LCSP solvent for polyethylene.

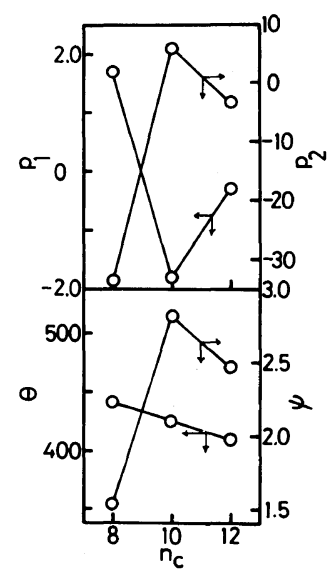

Figure 6. $p_{1}, p_{2}, \theta$, and $\psi$ plotted against the carbon number $n_{\mathrm{c}}$ constituting linear aliphatic hydrocarbons as UCSP solvent for polyethylene. 


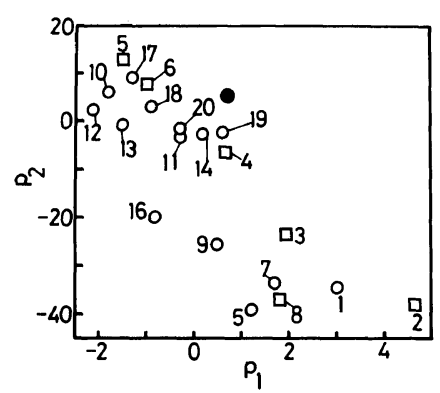

Figure 7. Plot of $p_{1}(\mathrm{KM})$ versus $p_{2}(\mathrm{KM})$ for polyethylene solutions: rectangle, LCSP; unfilled circle, UCSP; filled circle, the point theoretically expected when $A_{2}=A_{3}=A_{4}=0$ at $\theta$ : Number has the same meaning as those in Figure 3.

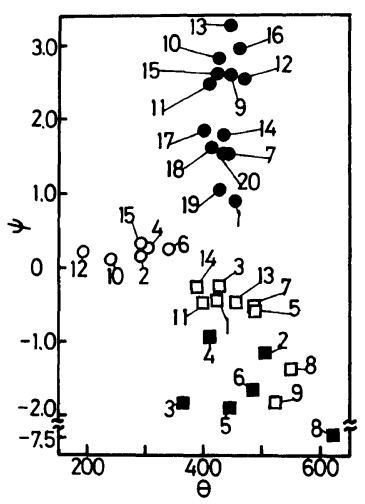

Figure 8. Plot of $\psi$ as a function of $\theta$ for polystyrene (unfilled mark) and polyethylene (filled mark) solutions: circle, UCSP; rectangle, LCSP. Number has the same meaning as those in Figures 1 and 3.

tem is concerned, we can obtain $p_{1} \cong 2 / 3$ as in the case of PS/solvent systems.

Figure 5 shows the plot of $p_{1}, p_{2}, \theta$, and $\psi$ as a function of the carbon number $n_{\mathrm{c}}$ constituting linear aliphatic hydrocarbons as LCSP solvent systems investigated. In short, increase in $n_{\mathrm{c}}$ and then slowly increases after passing through minimum at $n$-heptane. $n_{\mathrm{c}}$ dependence of $p_{2}$ is just reverse to that of $p_{1}$. Although $\psi$ does not show a systematic change with $n_{\mathrm{c}}, \theta$ increases with $n_{\mathrm{c}}$.

Figure 6 demonstrates the plot of $p_{1}, p_{2}, \theta$, and $\psi$ against the carbon number $n_{\mathrm{c}}$ constituting linear aliphatic alcohols as UCSP solvent for PE. $p_{1}$ shows maximum and both $p_{2}$ and $\psi$ attain maximum at $n_{\mathrm{c}}=10$ (i.e., $n$-decyl alcohol). $\theta$ decreases linearly with $n_{\mathrm{c}}$.

Figure 7 illustrates the correlationship between $p_{1}$ and $p_{2}$, both estimated by KM method, for $\mathrm{PE}$ solutions. The theoretical point predicted where $A_{2}=A_{3}=A_{4}=0$ at $\theta$ temperature is denoted as a filled circle. It is interesting to note that, unlike PS solutions, $p_{1}$ cannot be regarded as nearly constant and $p_{2}$ has a tendency to decrease with an increase in $p_{1}$, satisfying $p_{1} p_{2} \lesssim 0$.

Figure 8 shows the correlationships between $\psi$ and $\theta$ of PS (unfilled mark) and PE (filled mark). For PS solutions, the solvent dependence of $\psi$ is much smaller than that of $\theta$ in the both ranges of UCSP and LCSP. In contrast to this, for UCSP of PE solutions $\psi$ variation with solvent is remarkable as compared with the solvent dependence of $\theta$. The difference in $\psi$ between UCSP and LCSP is very significant in $\mathrm{PE}$ solution and the following relations holds:

$$
\psi_{\mathrm{PE}}^{\mathrm{UCSP}}>\psi_{\mathrm{PS}}^{\mathrm{UCSP}}>\psi_{\mathrm{PS}}^{\mathrm{LCSP}}>\psi_{\mathrm{PE}}^{\mathrm{LCSP}}
$$

For convenience, we express the Flory temperature evaluated by KM, KKS, and SF methods as $\theta(\mathrm{KM}), \theta(\mathrm{KKS})$, and $\theta(\mathrm{SF})$, respectively. For the UCSP of PS/single solvent system, $\theta(\mathrm{SF})$ is on the average $c a .1 .8 \mathrm{~K}$ higher than $\theta(\mathbf{K M})$ and $\theta(\mathbf{K K S})$. For LCSP of the same system $\theta(\mathrm{SF})$ is $2-3 \mathrm{~K}$ lower than $\theta(\mathrm{KM})$ and $\theta(\mathrm{KKS})$. For the $\mathrm{PE} /$ single solvent system $\theta(\mathrm{SF})$ is $c a .3-4 \mathrm{~K}$ higher in UCSP and $c a .8 \mathrm{~K}$ lower in LCSP than $\theta(\mathrm{KM})$ and $\theta(\mathrm{KKS})$. The difference between $\theta(\mathrm{KM})$ and $\theta(\mathrm{KKS})$ is practically insignificant for all the polymersolvent systems invenstigated. In short, $\theta(\mathrm{KM}) \cong \theta(\mathrm{KKS})<\theta(\mathrm{SF})$ for UCSP and $\theta(\mathrm{KM}) \cong \theta(\mathrm{KKS})>\theta(\mathrm{SF})$ for LCSP. The Flory temperature is believed the most reliable when it is determined as the temperature at which the second virial coefficient $A_{2}$ by the membrane osmometry or the light scattering method becomes zero (hereafter referred to as $\left.\theta\left(A_{2}\right)\right)$. $\theta\left(A_{2}\right)$ was found to be in the range 307.0 and $308.4 \mathrm{~K}$ (Table II of ref 2) and averaged to $307.6 \mathrm{~K}$ for UCSP 
of $\mathrm{PS} / \mathrm{CH}$ system. For this system, $\theta(\mathrm{KM})$, $\theta(\mathrm{KKS})$ and $\theta(\mathrm{SF})$ are by $2.5,2.4$, and $1.1 \mathrm{~K}$ underestimated. $\theta\left(A_{2}\right)$ was determined for LCSP of PS/MCH system to be 340.4 and $341 \mathrm{~K}$ (Table IV of ref 2) and averaged to be $340.7 \mathrm{~K}$ which is 0.5 and $1.1 \mathrm{~K}$ higher than $\theta(\mathrm{KM})$ and $\theta(\mathrm{KKS})$ and $1.6 \mathrm{~K}$ lower than $\theta(\mathrm{SF})$. From a theoretical point of view, KM and KKS methods are superior as compared with SF method. But, to estimate $\theta$ more accurately using $\mathrm{KM}$ or $\mathrm{KKS}$ method, the temperature dependence of $p_{1}$ and $p_{2}$ should be taken into account.

Summarizing, literature data on the cloud point curve and the critical solution point for PS solutions are accurate enough to be analyzed by KKS and KM methods and $p_{1}$ was found to be fairly near to the theoretical value (2/3), expected when $A_{2}=A_{3}=0$ at $\theta$. However, $p_{2}$ for these polymer/solvent systems deviates occasionally to a large extent, from the theoretical value $(1 / 2)$, calculated when $A_{2}=A_{3}=A_{4}=0$ at $\theta$ and $p_{2}$ is positive for UCSP and negative for LCSP. This suggests that the fourth virial coefficient $A_{4}$ does not always become zero even at $\theta$. The corresponding literature data for PE solutions are unfortunately rather qualitative and more reliable data for this polymer is highly anticipated. The entropy parameter $\psi$ for PS and PE solutions is negative at the LCSP region and positive at the UCSP region and an athermal solution will be realized, at least, for these polymers in any solvent at a specific temperature between LCSP and UCSP.

\section{REFERENCES}

1. R. Koningsveld, L. A. Kleintjens, and A. R. Shultz,
J. Polym. Sci., A-2, 8, 1262 (1970).

2. K. Kamide and S. Matsuda, Polym. J., 16, 807 (1984).

3. K. Kamide, S. Matsuda, T. Dobashi, and M. Kaneko, Polym. J., 16, 821 (1984).

4. A. R. Shultz and P. J. Flory, J. Am. Chem. Soc., 74, 4760 (1952).

5. W. R. Krigbaum, J. Am. Chem. Soc., 76, 3758 (1954).

6. K. Kamide, Y. Miyazaki, and T. Abe, Polym. J., 14, 355 (1982).

7. P. J. Flory, "Principle of Polymer Chemistry," Cornell Univ. Press, Ithaca, 1953.

8. M. Kurata, "Thermodynamics of Polymer Solutions," Harwood Academic Pubs. Chur, London, New York, 1982, Chapter 2.

9. R. Koningsveld and L. A. Kleintjens, Macromolecules, 5, 637 (1971).

10. N. Kuwahara, M. Nakata, and M. Kaneko, Polymer, 14, 415 (1973).

11. S. Saeki, N. Kuwahara, S. Konno, and M. Kaneko, Macromolecules, 6, 246 (1973).

12. T. Dobashi, M. Nakata, and M. Kaneko, J. Chem. Phys., 72, 6685 (1980).

13. S. Saeki, N. Kuwahara, S. Konno, and M. Kaneko, Macromolecules, 6, 589 (1973).

14. S. Saeki, S. Konno, N. Kuwahara, M. Nakata, and M: Kaneko, Macromolecules, 7, 521 (1974).

15. K. S. Siow, G. Delmas, and D. Patterson, Macromolecules, 5, 29 (1972).

16. M. Nakata, S. Higashida, N. Kuwahara, S. Saeki, and M. Kaneko, J. Chem. Phys., 64, 1022 (1976).

17. R. Koningsveld and A. J. Staverman, J. Polym. Sci., $A-2,6,325$ (1968).

18. N. Kuwahara, S. Saeki, T. Chiba, and M. Kaneko, Polymer, 15, 777 (1974).

19. F. Hamada, K. Fujisawa, and A. Nakajima, Polym. J., 4, 316 (1973).

20. A. Nakajima, H. Fujiwara, and F. Hamada, $J$. Polym. Sci., A-2, 4, 507 (1966).

21. Y. Muraoka, H. Inagaki, and H. Suzuki, Br. Polym. J., 15, 110 (1983).

22. A. Nakajima, F. Hamada, and S. Hayashi, J. Polym. Sci., C, 15, 285 (1966).

23. D. Patterson and G. Delmas, Trans. Faraday Soc:, 65, 708 (1969).

24. P. J. Flory, Discuss. Faraday Soc., 49, 7 (1970). 